\title{
Molecular Biomarkers according to Primary Tumor Location in Colorectal Cancer: Current Standard and New Insights
}

\author{
Gianluigi De Renzi Giulio Gaballo Paola Gazzaniga Chiara Nicolazzo \\ Department of Molecular Medicine, Cancer Liquid Biopsy Unit, Sapienza University of Rome, Rome, Italy
}

\section{Keywords}

Colorectal cancer · Biomarkers · Sidedness · Precision

medicine $\cdot$ Liquid biopsy

\begin{abstract}
According to the 2018 GLOBOCAN database, colorectal cancer is one of the most common malignancies and leading causes of cancer-related death worldwide. During the last decades, considerable progress has been made in understanding the complex pathogenetic mechanisms involved in this neoplastic disease. Due to the need to improve treatment responses and clinical outcomes of colorectal cancer patients, the identification of new molecular biomarkers became a crucial spot in clinical oncology. As biological indicators of a specific pathological or physiological process, molecular markers play a central role in cancer detection, diagnosis, outcomeprediction, and treatmentchoice.Considering the existing evidence that malignancies originating from distinct colonic regions behave differently, it is clear that specific biomarkers can be associated to right- or left-sided colon carcinomas, reflecting the distinct molecular signatures of these different tumor entities. The aim of this review is to summarize the main differences among tumors arising from proximal and distal colon in terms of current and emerging biomarkers.

(c) 2020 S. Karger AG, Basel
\end{abstract}

\section{Introduction}

Colorectal cancer (CRC) is one of the most common malignancies and leading causes of cancer-related death worldwide. According to the GLOBOCAN database, it has been estimated that nearly $1,850,000$ new cases were diagnosed and 880,000 people died in 2018 [1]. During the last decades, considerable progress has been made in understanding the complex pathogenetic mechanisms involved in this neoplastic disease. CRC development usually occurs in the lining of the colon or rectum. Several studies report that generally colon cancer evolution begins from benign or non-cancerous forms, called polyps. When a polyp is not treated or removed, it can become a potentially lethal tumor. With this in mind, the earlier the polyps are detected, the greater the chances of preventing CRC [2]. Clinical management of patients with advanced CRC is clearly more difficult because of the continuous development of this disease. The spatial and temporal evolution of cancer perfectly describes the dynamic nature of colorectal tumors and offers a clear picture of their complexity and heterogeneity [3]. Due to the need to improve screening, prevention, treatment responses, and clinical outcomes of CRC patients, the identification of new molecular biomarkers became a crucial spot in clinical oncology. As biological indicators of a specific pathological or phys- karger@karger.com

www.karger.com/ocl

Karger ${ }^{\prime}=$
(C) 2020 S. Karger AG, Basel
Chiara Nicolazzo

Department of Molecular Medicine, Sapienza University of Rome

Viale Regina Elena, 324

IT-00161 Rome (Italy)

chiara.nicolazzo@uniroma1.it 
iological process, molecular markers serve as precious tools for cancer detection, diagnosis, patient prognosis, and treatment choice [2]. To date, differences among CRCs in terms of patient, histological and microbiome characteristics, diet, and molecular alterations have been observed [4]. Fairly recent studies report indeed that there is not a random distribution, but a close association of these features based on primary tumor location [4]. Colon carcinomas are generally defined right-sided when they originate from proximal colonic regions up to the splenic flexure, whilst descending colon gives rise to left-sided colon ones. The use of the splenic flexure as a division line between proximal and distal colon is determined by the fact that the first two thirds of the transverse colon up to the splenic flexure embryologically derive from the midgut, while the distal third arises from the hindgut. These two embryological origins may underlie the differences between right and left colon cancers $[4,5]$. Furthermore, right-sided tumors occur with lower incidence and worse prognosis mostly in older-aged female subjects. They are characterized by a flat-like morphology with mucinous, undifferentiated, or signet-ring cell histology and they are more likely to metastasize to the peritoneum [4-6]. Proximal colon cancers are also most associated with microsatellite instability (MSI)-high (MSI-H) status, which makes them highly immunogenic tumors, with a $\mathrm{CpG}$ island methylator phenotype (CIMP)-high status and BRAF mutations [4-7]. On the other hand, the incidence of tumors arising from distal colon is higher than right-sided ones and the probability of a left-sided carcinoma is greater in men [4]. Left-sided colon cancers show a polypoid-like morphology and a marked tendency to metastasize in liver, lungs, or, less commonly, in bones [4-6]. The main molecular characteristics of distal colonic cancers include chromosomal instability (CIN), CIN pathway-related mutations, such as APC, SMAD4, and TP53, together with overexpression of the epidermal growth factor receptor (EGFR) ligands $[4,6]$. Thus, considering the existing evidence that malignancies originating from distinct colonic regions behave differently, it is clear that specific biomarkers can be associated to right- or left-sided colon carcinomas, reflecting the distinct molecular signatures of these different tumor entities $[4,7]$. The first part of the review is intended to be a description of the main characteristics of molecular biomarkers used in daily clinical practice, with reference to their prognostic and predictive value and their association with the primary location of the tumor. The second part is instead focused on the scenario of emerging biomarkers in terms of the differences between right-sided and left-sided colon cancers.

\section{Current Molecular Biomarkers by Side: Is What We Have Enough?}

However broad the molecular landscape related to both left- and right-sided colon cancers is, those that play a leading role in current clinical practice, serving either as prognostic or predictive biomarkers, are the mutational status of RAS and BRAF genes along with mismatch repair (MMR) gene deficiency (dMMR). Numerous studies were performed by using such markers and their analyses are highly recommended to gain information for patient risk stratification and to optimize therapeutic choices [7-9].

\section{RAS}

Solid scientific evidence describes the pivotal role of mutations in genes of the RAS family, such as KRAS, NRAS, and HRAS in different tumor types, including CRCs. Kirsten RAS gene mutations are the most representative in CRC: about a third of CRCs shows hotspot mutations in KRAS [7]. These mutations are commonly associated with right-sided colon carcinomas and their expression progressively decreases from proximal to distal regions of the colon [7]. Although the prognostic significance of KRAS mutations in both primary and metastatic colon cancers has been extensively discussed in the last decades, conflicting evidence exists about the implications of KRAS alterations on CRC patients' survival [10-13]. On the other hand, NRAS mutations are less frequent in CRC patients. Interestingly, it has been observed that NRAS-mutated CRCs are associated with a worse prognostic impact than KRAS-mutated CRCs [14]. However, mutations of members of the RAS gene family lead to a constitutive activation of the RAS-RAF-MEK-ERK-MAP kinase pathway, involved in cell growth, proliferation, and differentiation and are mutually exclusive to mutations of BRAF and other components of the MAP kinase cascade $[7,8]$.

The current clinical guidelines highly recommend testing the mutational status of RAS in the case of patients with metastatic CRC (mCRC) since the presence of mutations in RAS genes indicates a non-response to antiEGFR treatments, although its prognostic value is still debatable [15]. Nevertheless, it has been observed that less than half of patients with wild-type (wt) status of RAS respond to anti-EGFRs [7]. A recent retrospective analysis showed that the primary tumor localization significantly influences the response to anti-EGFR in the case of metastatic RAS-wt tumors [16]. Left-sided colon cancers were found to better respond to chemotherapy plus antiEGFR than to chemotherapy alone or chemotherapy plus bevacizumab. Thus, tumors of the right side seem not to 
benefit from EGFR inhibition. Furthermore, the failure of anti-EGFR treatments might be due to a form of primary or secondary resistance. The mechanisms underlying primary resistance seem to involve the amplification of MET and HER2, phosphatidylinositide-3-kinase (PIK3CA), FGFR1 and PDGFRA mutations, low EGFR copy number, and loss of PTEN function [17]. Secondary resistance instead is strictly related to the evolution of resistant clones induced by anti-EGFRs selective pressure [3]. Hence the importance of liquid biopsy in monitoring disease progression.

\section{$B R A F$}

RAF is another core component in the RAS signaling pathway which affects cell growth, proliferation, and differentiation, but RAF implication in regulating apoptosis, cell migration, and survival has also been reported [8]. BRAF mutations are found in up to $10 \%$ of CRCs and several studies showed their prevalence in right-sided ones along with their association with MSI $[7,8]$. In addition, such mutations lead to the constitutive activation of the EGFR downstream signaling pathway and, since they participate in the same signaling cascade, BRAF and RAS mutations are considered mutually exclusive $[7,8$, 15]. The most representative BRAF mutation is certainly the V600E, which involves the replacement of a glutamic acid for a valine at residue 600 (V600E) within the protein kinase domain [8].

The negative prognostic impact of BRAF V600E mutation in $\mathrm{mCRC}$ patients has been assessed in multiple studies $[7,8,18,19]$. Furthermore, the prognostic value of BRAF takes on a particularly negative significance when associations between MSI-H tumors and BRAF V600E mutation are found. About $60 \%$ of MSI-H colon cancers are enriched with BRAF V600E mutation [7]. It has also been found that patients with other BRAF mutations are often associated with microsatellite stability and markedly longer overall survival (OS), compared with BRAF V600E CRC patients [20].

In addition to its prognostic significance, BRAF-mutated status is also predictive of unresponsiveness to antiEGFR therapies [7-9, 19]. A recent metanalysis showed no increase of benefit from anti-EGFRs compared to standard therapy in RAS-wt/BRAF-mutated CRC patients, confirming the predictive significance of BRAF mutations [21].

\section{MSI}

MSI is the condition of genetic hypermutability that results from impaired DNA MMR. In sporadic tumors, hypermethylation of the MLH1 gene promoter, involved in MMR, is the primary cause of MMR deficiency. On the other hand, germline mutations of MMR genes might be the cause of the MSI-H status and they might be responsible for hereditary nonpolyposis CRC. It is possible to distinguish sporadic MSI from hereditary nonpolyposis CRC by the simultaneous presence of a CIMP and BRAF V600E mutation in MSI-H CRC tumors [7, 8]. MSI-H tumors are mainly associated with certain clinicopathological features, including primary tumor location: MSI$\mathrm{H}$ CRCs are typically right-sided $[7,8]$. Moreover, it has been observed that approximately $15 \%$ of primary CRCs are MSI-H, while the MSI-H status is less common among patients with metastatic disease (reported in $\leq 5 \%$ of patients with mCRC) [7].

In the case of primary CRCs, MSI status is widely accepted as a positive prognostic factor $[7,8]$. This favorable prognostic association might be biologically justified by the high tumor molecular burden caused by impaired MMR. High tumor molecular burden leads indeed to the formation of neoantigens, thus causing a strong antitumor immune response [7]. Sinicrope et al. [22] confirmed the prognostic impact of dMMR, underlining the association between a favorable outcome and the proximal localization of dMMR tumors $(p=0.018)$. Concerning patients with metastatic disease, the MSI-H status is indicative of poor prognosis instead [7]. Several studies report that median progression-free survival (PFS) and OS were significantly worse for patients with dMMR compared with proficient MMR tumors [23]. The reasons for this paradoxical effect are not entirely clear. It has been proposed that the enrichment with BRAF mutations could provide an explanation for the aggressiveness of metastatic MSI-H tumors [7].

Furthermore, the predictive value of MSI status has been assessed for both primary CRC and $\mathrm{mCRC}$ and it is currently useful in clinical practice. According to the recent guidelines, the presence of MSI in early CRC stages has been reported to be predictive for non-response to 5-fluorouracil (5-FU)-based adjuvant chemotherapy. On the other side, emerging data show a potential benefit for metastatic MSI tumors from the treatment with immune checkpoint inhibitors (ICIs) [15]. It is possible to apply the reason for the positive prognostic value exerted by the MSI-H state in primary CRCs to explain the sensitivity to ICIs [7]. As previously reported, the hypermutated status of MSI-H tumors results in the generation of neoantigens, which leads to antitumor immune response. This defensive mechanism is secondarily responsible for the generation of an immunosuppressive tumoral environ- 
ment, due to the upregulation of both programmed death-ligand 1 (PD-L1) in cancer cells and immunomodulatory receptors on $\mathrm{T}$ cells, which mediate the immune response [7]. Because of the interaction between these two counterparts, cancer cells can evade the immune surveillance [7]. By inhibiting this interaction, the ICIs currently approved for the treatment of these mCRCs, i.e., pembrolizumab, nivolumab (anti-programmed death-1 [PD-1]), and ipilinumab (anti-CTL4), enable the reactivation of the antitumor immune response [7-9].

It is evident that the number of biomarkers currently available in clinical practice is considerably limited compared to the complexity of CRC. Certainly, high hopes are pinned on the identification and validation of new molecular markers to improve the management of oncologic patients.

\section{Emerging Biomarkers}

\section{Biomarkers Associated with Left-Sided Colon Cancer}

KISS1 gene codes for kisspeptins, a family protein in which kisspeptin 54, kisspeptin 14, kisspeptin 13, and kisspeptin 10 are involved; these proteins bind to KISS1R, a q/11 GPCR. In cancer patients, kisspeptins regulate the metastatic potential of malignant cells by inhibiting migration and metastasis and the infiltration of underlying tissues, suppressing angiogenesis and cell proliferation, and they can induce apoptosis as well [24]. Although the highest expression has been found in healthy subjects, cancer patients' high expression of KISS1 leads to poor prognosis. In addition, although some studies admit that kisspeptin levels are higher in early rather than in late stages, and other studies assert the contrary, Kostakis et al. [24] found that high KISS1 levels belong to III/IV stages and tumors larger than $4 \mathrm{~cm}$, as well as lymph node metastatic diseases. Patients with low KISS1 levels show better values of disease-specific survival and recurrence-free survival (RFS) as opposed to patients with high expression levels. Noteworthy, KISS1 expression levels, in healthy subjects, turned out to be higher in right-sided colon $(p=$ 0.016 ), while in tumor patients they are higher in left-sided cancer $(p=0.049)$ [24]. Finally, Kostakis et al.'s study suggests that KISS1 expression is downregulated during metastasis, but in advanced stages (III/IV) and in tumors more than $4 \mathrm{~cm}$ in size, KISS1 levels return to high. Lower levels of KISS1R suggest that there may be a different binding site of KISS1 [24].

Src belong to non-receptors cytoplasmic tyrosine-kinase family protein, and it is mainly activated by stress and growth factors. Src is involved in MAPK/ERK pathway, PI3K/AKT pathway, SRC/FAX pathway, and $\beta$-catenin/E-cadherin complex, facilitating their activation, being thus involved in metastatic and staminal tumor patterns; its involvement results also in invasion, proliferation, adhesion, migration, and differentiation mechanisms. Src activity also confers resistance to platinum-based drugs, suggesting that it may be considered as predictive biomarker. High expression levels of Src are associated to poor prognosis (regarding neoadjuvant therapy) and with poor 5-year PFS; it is not possible to assert the same for the OS rate. These features are correlated with left-sided colon cancers $(p<0.001)$ [25].

$c$-MET is a proto-oncogene that encodes the tyrosinekinase receptor for the hepatocyte growth factor/scatter factor (HGF/SF). The interaction between $\mathrm{c}-\mathrm{MET}$ and HGF/SF contributes to tumor aggressiveness promoting cell proliferation, angiogenesis, survival, migration, and metastasis. The c-MET aberrant activity is due to point mutations, gene amplification, transcriptional upregulation, or ligand autocrine loops of its gene [26]. Although more studies are needed, high c-MET levels seem to be correlated to left-sided colon cancer $(p<0.05)$, tumor size larger than $5 \mathrm{~cm}$, to advanced TNM stages, and with nodal metastasis. c-MET overexpression has also been correlated to local tumor recurrence after preoperative chemotherapy. Furthermore, high c-MET expression levels have been associated to neo-angiogenesis process, since this tyrosine-kinase receptor can increase the endothelial cell growth and the expression of pro-angiogenic vascular endothelial growth factor, inhibiting thrombospondin-1, a negative angiogenesis regulator [26].

Erythropoietin-producing hepatocellular A2 (EPHA2) is a tyrosine-kinase receptor involved in cancer cell growth, migration, and invasiveness through different pathways such as RAS and AKT, epithelial-mesenchymal transition (EMT) process, and integrin-mediated adhesion, and high levels of EPHA2 expression have been found at the invasive tumor front, compared to the center of the tumor [27]. EPHA2 expression has been evaluated in RAS/BRAF-wt patients in treatment with cetuximabFOLFIRI first-line therapy; its overexpression has been correlated to reduced treatment efficacy. EPHA2 has been found overexpressed in early tumor stages (I-II), and it has also been associated with poor prognosis in IIIII stages. Regarding the sidedness, high expression levels have been found in less differentiated left-sided colon cancer $(p=0.04)$. Patients with high EPHA2 expression levels have shown shorter PFS compared to patients with low EPHA2 expression [27]. 
Leucine-rich repeat containing G-protein-coupled receptor 5 (LGR5) is a receptor of R-spondins, in the Wnt signaling pathway. It is an adult stem cell marker in healthy subjects [28]. Although several studies correlated high LGR5 expression levels to poor prognosis, Jang et al. [28] showed that higher expression of LGR5 is correlated to better prognosis, in terms of RFS and OS. Although LGR5 is an adult stem cell marker, the correlation between this marker and the most common cancer stem cell markers has been reported as not significant [28]. LGR5 has been correlated with left-sided colon cancer $(p=$ 0.019), microsatellite stability, CIMP-negative profile, $\mathrm{CIN}$, and nuclear $\beta$-catenin expression leading to an abnormal Wnt signal activation, features that result more evident in stage III CRC patients than in stage I, II, or IV [28]. Therefore, although LGR5 has different roles (enhancer or suppressor) based on cancer site, in CRC this receptor is an independent good prognostic marker, acting as tumor suppressor, attenuating tumor growth and migration.

Hippo pathway controls the physiological tissue growth, then an alteration of Hippo pathway may lead to tumor growth and proliferation. Regarding this signaling pathway, YAP and TAZ may be considered among the main factors involved in activating metastatic process. These factors are degraded by a pool of proteins that constitute the core of Hippo pathways (MST1, MST2, LATS1, LATS2, MOB1A, MOB1B) [29]. If this signaling pathway is inactivated, YAP and TAZ induce nuclear activation of the gene involved in stem cell maintenance, EMT program, and metastasis development. Hippo pathway, through YAP and TAZ, collaborates with Hedgehog, Notch, and TGF- $\beta$ pathways and its inhibition results in RASSF1a methylation [29].

In high-risk stage II/III CRC the 3-year recurrence rate is correlated with RASSF1a, TAZ, and MST1 polymorphism in patients undergoing 5-FU-based chemotherapy. Noteworthy, TAZ-related rs3811715 polymorphism is correlated to left-sided tumors $(p=0.011)$ and to CT or TT genotypes. In addition, in left-sided tumors the MST1related rs 17420378 polymorphism is also associated with recurrence probability [29].

HER2 is a tyrosine-kinase receptor, member of the EGFR family proteins. The role of HER2 expression as CRC biomarker is not clearly defined to date; nevertheless, some studies showed that amplified HER2 may induce chemoresistance in patients refractory to anti-EGFR therapy $[19,30]$. Another study shows that RAS/RAF-wt CRC may be characterized by HER2 amplification. Furthermore, the amplification of HER2 in patients with stage II/III rectal cancer undergoing second- or third-line anti-EGFR therapy, may lead to a significantly shorter PFS compared to patients with HER2-negative tumors [31]. Thus, HER2 might be defined as a negative prognostic biomarker.

In addition, a study conducted by Nam et al. [32] has shown that HER2 expression is higher in left-sided compared to right-sided colon cancers $(p=0.006)$.

\section{Biomarkers Associated with Right-Sided Colon Cancer}

The above-mentioned Hippo pathway is also linked to right-sided colon cancer, through RASSF1-related rs2236947 polymorphism, representing the most important factor to predict recurrence probability. To date, polymorphisms located in genes involved in this pathway have never been evaluated as biomarkers for colon cancer [29].

RET gene encodes for several tyrosine-kinase receptors proteins. Taken individually, they do not have a predictive or prognostic role. However, if a rearrangement of these genes occurs, the RET fusion becomes predictive and prognostic. RET rearrangement is involved in PI3K/ Akt and JAK/STAT pathways, suggesting an involvement in both metastasis and invasion programs [33]. RET fusion (NCOA4-RET and CCDC6-RET genes mainly) mostly occurs in aged patients, with a 1-2 performance status, in right-sided colon cancer $(p=0.013)$ and in nonresected primary tumors. Other important tumor features correlated to RET fusion are the MSI-H profile and the RAS/BRAF-wt status [33].

MUC16 is a human glycoprotein, member of the mucin family glycoproteins. In the colon environment, since mucin is highly glycosylated, it creates a viscous protective layer on the apical membrane of epithelial cells, protecting the colon against infectious agents. C-terminus of MUC16 activates the Wnt signaling pathway through its interaction with $\beta$-catenin, which facilitates its transport into the nucleus, allowing metastasis and invasiveness. The high expression of MUC16 in patients with rightsided colon cancer determines poor prognosis $(p<0.001)$, especially if associated with lower expression of EGFR and large tumor size. MUC16 has also been described to have a negative predictive role in cisplatin-based therapy [34].

KLK13 is a member of the kallikreins family, a subgroup of serine proteases. Low levels of KLK13 in rightsided compared to left-sided cancer patients determines poor prognosis $(p=0.001)$ [34].

HES1 is a downstream target of the Notch pathway. When Notch signaling pathway is activated, there is an 
increased expression of HES1. A study conducted by Ahadi et al. [35] found that the loss of nuclear expression of HES1 is higher in right colon cancer $(p<0.001)$, in aged patients, and in high histologic grade, and it is correlated with MSI-H status, BRAF V600E mutation, and large tumor size. The loss of nuclear expression of HES1 determines a poor 5-year OS [35].

The downregulation of zymogen granule 16 protein (ZG16) plays a role in CRC; indeed, cancer patients show low ZG16 expression levels compared to healthy subjects. Cancer cells are able to avoid the immune system binding some portions of carbohydrates. Carbohydrates play a role in the metastasis, being involved in tumor celltumor cell adhesion, tumor cell-extracellular membrane adhesion, and tumor cell-endothelial cell adhesion. Carbohydrates can also worsen inflammatory states. Lectines are involved in the metastatic process by binding carbohydrates as well. Conversely, lectines not linked to carbohydrates are implicated in the first-line defense against pathogens, immune regulation, and prevention of autoimmunity. Jacaline, a member of the lectines family, has some important features like T-cell stimulation, recognition, and tumor cell inhibition. Sequence analysis showed that the ZG16 protein contained a jacaline-like lectin domain that confers to ZG16 protein the abovementioned jacaline features. Thus, loss of ZG16 determines a poor prognosis in colon cancer. High levels of ZG16 have been found in descending colon compared to ascending colon $(p=0.02)$. High ZG16 expression is also associated with MSI-low compared to MSI-H tumors. Low levels of ZG16 expression have been correlated to hypermutated tumors and with silenced MLH1 expression and are associated with poor prognosis and short RFS [36].

The expression of both PD-1 and PD-L1 has been mostly associated with $\mathrm{dMMR}$, and, then, MSI-H tumors. In addition, high PD-1 expression on tumor-infiltrating lymphocytes (TILs) is associated with better RFS, while patients with high expression of both PD-1 and PD-L1 on TILs have a definitely poor prognosis [37].

There is a statistical difference between low and high expression of PD-1/PD-L1 on TILs in right-sided compared to left-sided colon cancers. Right-sided tumors are characterized by higher levels of both PD-1 and PD-L1, while left-sided ones are correlated to lower levels of both PD-1 and PD-L1. High levels of PD-1-positive TILs are associated with better prognosis in $\mathrm{dMMR}$ patients if $\mathrm{PD}$ L1 levels are low. When it is highly expressed, a poor RFS occurs. Also, high expression of both PD-1 and PD-L1 is frequent in dMMR tumors [37]. Wyss et al. [38] showed that PD-L1 expression is highly associated with right-sided colon cancer $(p=0.0051)$, female gender, older age, lymph node metastasis, higher tumor grade, and frequent positivity of lymphatic and venous vessels [17]. Despite these aggressive tumor features, the correlation between PD-L1 expression and MSI-H tumors determines improved disease-free survival [38].

ALDH1A1 is a member of the ALDH family proteins. Its main role is the detoxification of exogenous and endogenous aldehydes through a $\mathrm{NAD}(\mathrm{P})+$-dependent oxidation. The ALDH1 isoform has been found to be involved in cancer stem cells maintenance and survival, protecting them against reactive oxygen species. ALDH1A1 expression is associated with chemoresistance and radioresistance in CRC. Regarding sidedness, high expression levels of ALDH1A1 have been found in rightsided compared to left-sided colon cancers $(p=0.007)$ [39].

CIMP could be considered as an early tumor-initiating event. CIMP is a subset of colon cancer in which the CpG islands are highly methylated, leading cytosine to be 5-methylcytosine. TET family proteins (TET1, TET2, and TET3) catalyze the conversion from 5-methylcytosine to 5-hydroxymethylcytosine, leading to the demethylation process [40].

In CIMP-positive tumors, particularly those carrying BRAF mutation, TET1 gene is frequently silenced by DNA methylation, leading to tumor growth. TET1 methylation is correlated to metastasis, and the more TET1 is methylated, the less metastatic lesions occur. TET1 methylation is strictly correlated to the proximal colon $(p<$ $0.001)$ [40].

The tyrosine-kinase receptors ALK and ROS and the cytoplasmic non-receptor tyrosine-kinase NTRK rearrangements frequently occur in older patients with right-sided colon cancer $(p<0.001)$. Such rearrangements are also correlated to lymph node metastasis and MSI-H profiles. Regarding RAS/BRAF mutational status, ALK, ROS, and NRTK rearrangements are often correlated to a wt status of KRAS. Gene rearrangement is also correlated to poor OS, independently from sidedness and MSI status [41]. Despite the mutual exclusivity between BRAF mutations and ALK, ROS, and NRTK rearrangements, rearranged tumors are often correlated to RNF43 frameshift mutations [41]. Ring finger protein 43 (RNF43) is an E3 ubiquitin ligase protein involved in the Wnt pathway regulation. Since it degrades frizzled receptors (on which Wnt binds to start the signaling pathway that leads to EMT and metastasis gene transcription) and LRP5/6 factor, a wt 
form of RNF43 inhibits this pathway. Conversely, mutated RNF43 could lead to the accumulation of Wnt, leading to the activation of the $\mathrm{Wnt} / \beta$-catenin pathway. RNF43 is frequently mutated in BRAF-mutant diseases (BRAF mutation status is mutually exclusive with APC mutation status), and if RNF43 is downregulated, the highest tumoral growth has been observed in APC mutant cancers. RNF43 mutations were predominantly detected in MSI-H CRC in the right colon $(p<0.0001)$. RNF43 activity is inhibited by binding of R-spondins to Lgr4, Lgr5, or Lgr6, since this binding determines an accumulation of frizzled receptors [42].

The loss of the polycomb group protein enhancer of zeste homolog 2 (EZH2) has been associated with cancer growth and poor prognosis, since it determines the acquisition of mesenchymal features in the invasive front, leading to tumor cell dissemination and metastasis formation. In advanced tumor stages (III-IV, N1/N3 e/o M1) low levels of EZH2 are expressed, with high EZH2 expression being associated with poor prognosis [43].

EZH2 is a methyltransferase that lowers the miR-31 levels (miR-31 can regulate downstream the EGFR pathway) by methylating three times histone 3 lysine 27 (H3K27me3) on the miR-31 promoter. Since methylation is equivalent to gene silencing, high expression of EZH2 is correlated to low miR-31 levels [44]. Low EZH2 expression has been associated with short PFS rate, mainly in KRAS-wt tumors. Noteworthy, short PFS and OS rates have been correlated to low EZH2 expression levels, regardless of KRAS, NRAS, and BRAF mutations, suggesting that EZH2 expression is a useful negative predictive biomarker for anti-EGFR therapies. Regarding the sidedness, low EZH2 expression has been found in proximal colon cancer $(p=0.0082)$ [44].

\section{Liquid Biopsy-Based Biomarkers}

Liquid biopsy provides a noninvasive route of sample collection for analysis of circulating tumor cells (CTC) and cell-free DNA, with the advantage of longitudinal molecular characterization of the disease. Although it has been described that liquid biopsy-based assays are useful screening methods for both left- and right-sided colon cancers [45], few studies have been reported to date concerning the difference of liquid biopsy-based information according to tumor sidedness. A recent study evaluated whether the presence of CTC might dichotomize according to sidedness [46]. Authors described a phenotypic heterogeneity of CTC, with a predominant mesenchymal phenotype found in CTC from distal compared to proximal primary tumors hypothesizing that proximal and distal CRCs may adopt different modalities of tumor cells intravasation depending on primary tumor location. Interestingly, CTC isolated from right-sided tumors exhibited a clear apoptotic pattern, providing the rationale for the limited prognostic impact of these cells in patients with primary proximal cancers. Different somatic DNA alteration landscapes have been reported among the leftsided, right-sided, and rectal cancer, with right-sided colon cancer being associated with higher number of mutations in circulating tumor DNA than left-sided colon and rectal cancers [47].

\section{Conclusion}

Considering the high variability of clinical outcomes and treatment responses of CRC patients, it is clear that there is the need to identify new molecular biomarkers to guide oncologists in managing CRC patients and lead to increasingly personalized therapeutic approaches. It has been frequently observed that there is a strong association between molecular markers and the primary site of the tumor. In particular, the prognostic or predictive significance of a certain biomarker seems to reflect tumor characteristics, according to its primary location. For instance, BRAF mutations have a negative prognostic impact in patients with mCRC $[5,8]$. Also, BRAF-mutated tumors are often characterized by MSI-H status and show an aggressive behavior [5]. Both BRAF mutations and MIS-H status are strongly associated with cancers of the right side of the colon, which have a worse prognosis than those of the left side $[4-6,8]$. With this in mind, biomarker-guided therapy can certainly lead both to a considerable improvement in patients' survival or treatment response and to a progressively complex situation due to the increasing number of potential biomarkers and their tangled interactions. For this reason, it is important not only to simply identify new prognostic and predictive disease biomarkers, but also to understand their elaborate network. The broader use of liquid biopsy might allow in future to track tumor evolution throughout treatment leading to a more personalized approach in left- and right-sided CRCs.

\section{Conflict of Interest Statement}

The authors have no conflicts of interest to declare. 


\section{Funding Sources}

The authors received no specific funding for this work.

\section{Author Contributions} view.

\section{References}

1 Ferlay J, Colombet M, Soerjomataram I, Mathers C, Parkin DM, Piñeros M, et al. Estimating the global cancer incidence and mortality in 2018: GLOBOCAN sources and methods. Int J Cancer. 2019;144(8):1941-53.

2 Das V, Kalita J, Pal M. Predictive and prognostic biomarkers in colorectal cancer: A systematic review of recent advances and challenges. Biomed Pharmacother. 2017;87:8-19.

3 Siravegna G, Mussolin B, Buscarino M, Corti G, Cassingena A, Crisafulli G, et al. Clonal evolution and resistance to EGFR blockade in the blood of colorectal cancer patients. Nat Med. 2015;21(7):827-01.

4 Yang SY, Cho MS, Kim NK. Difference between right-sided and left-sided colorectal cancers: from embryology to molecular subtype. Expert Rev Anticancer Ther. 2018;18(4): 351-8.

5 Lee MS, Menter DG, Kopetz S. Right versus left colon cancer biology: integrating the consensus molecular subtypes. J Natl Compr Canc Netw. 2017;15(3):411-9.

6 Baran B, Mert Ozupek N, Yerli Tetik N, Acar E, Bekcioglu O, Baskin Y. Difference Between Left-Sided and Right-Sided Colorectal Cancer: A Focused Review of Literature. Gastroenterology Res. 2018;11(4):264-73.

7 Sveen A, Kopetz S, Lothe RA. Biomarkerguided therapy for colorectal cancer: strength in complexity. Nat Rev Clin Oncol. 2020; 17(1):11-32.

8 Schirripa M, Cohen SA, Battaglin F, Lenz HJ. Biomarker-driven and molecular targeted therapies for colorectal cancers. Semin Oncol. 2018;45(3):124-32.

9 Davis AA, Cristofanilli M. Detection of Predictive Biomarkers Using Liquid Biopsies. Am J Manag Care. 2018;24:107-17.

10 Ogino S, Meyerhardt JA, Irahara N, Niedzwiecki D, Hollis D, Saltz LB, et al. KRAS mutation in stage III colon cancer and clinical outcome following intergroup trial CALGB 89803. Clin Cancer Res. 2009;15(23):7322-9.

11 Dienstmann R, Mason MJ, Sinicrope FA, Phipps AI, Tejpar S, Nesbakken A, et al. Prediction of overall survival in stage II and III colon cancer beyond TNM system: a retrospective, pooled biomarker study. Ann Oncol. 2017;28(5):1023-31.

12 Saridaki Z, Tzardi M, Sfakianaki M, Papadaki C, Voutsina A, Kalykaki A, et al. BRAFV600E mutation analysis in patients with metastatic colorectal cancer (mCRC) in daily clinical practice: correlations with clinical characteristics, and its impact on patients' outcome. PLoS One. 2013;8(12):e84604.
13 Sjoquist KM, Renfro LA, Simes RJ, Tebbutt NC, Clarke S, Seymour MT, et al. Personalizing survival predictions in advanced colorectal cancer: the ARCAD nomogram project. J Natl Cancer Inst. 2018;110(6):63848.

14 Wang Y, Loree JM, Yu C, Tschautscher M, Briggler AM, Overman MJ, et al. Distinct impacts of KRAS, NRAS and BRAF mutations on survival of patients with metastatic colorectal cancer. JCO. 2018;36(15_Suppl 1): 3513.

15 Sepulveda AR, Hamilton SR, Allegra CJ, Grody W, Cushman-Vokoun AM, Funkhouser WK, et al. Molecular Biomarkers for the Evaluation of Colorectal Cancer: Guideline Summary From the American Society for Clinical Pathology, College of American Pathologists, Association for Molecular Pathology, and American Society of Clinical Oncology. J Oncol Pract. 2017;13(5):333-7.

16 Arnold D, Lueza B, Douillard JY, Peeters M, Lenz HJ, Venook A, et al. Prognostic and predictive value of primary tumour side in patients with RAS wild-type metastatic colorectal cancer treated with chemotherapy and EGFR directed antibodies in six randomized trials. Ann Oncol. 2017;28(8):1713-29.

17 Bertotti A, Papp E, Jones S, Adleff V, Anagnostou $\mathrm{V}$, Lupo $\mathrm{B}$, et al. The genomic landscape of response to EGFR blockade in colorectal cancer. Nature. 2015;526(7572): 263-7.

18 Yokota T, Ura T, Shibata N, Takahari D, Shitara K, Nomura M, et al. BRAF mutation is a powerful prognostic factor in advanced and recurrent colorectal cancer. Br J Cancer. 2011; 104(5):856-62.

19 Van Cutsem E, Cervantes A, Adam R, Sobrero A, Van Krieken JH, Aderka D, et al. ESMO consensus guidelines for the management of patients with metastatic colorectal cancer. Ann Oncol. 2016;27(8):1386-422.

20 Cremolini C, Di Bartolomeo M, Amatu A, Antoniotti C, Moretto R, Berenato R, et al. BRAF codons 594 and 596 mutations identify a new molecular subtype of metastatic colorectal cancer at favorable prognosis. Ann Oncol. 2015;26(10):2092-7.

21 Pietrantonio F, Petrelli F, Coinu A, Di Bartolomeo M, Borgonovo K, Maggi C, et al. Predictive role of BRAF mutations in patients with advanced colorectal cancer receiving cetuximab and panitumumab: a meta-analysis. Eur J Cancer. 2015;51(5):587-94.
22 Sinicrope FA, Mahoney MR, Smyrk TC, Thibodeau SN, Warren RS, Bertagnolli MM, et al. Prognostic impact of deficient DNA mismatch repair in patients with stage III colon cancer from a randomized trial of FOLFOX-based adjuvant chemotherapy. J Clin Oncol. 2013;31(29):3664-72.

23 Venderbosch S, Nagtegaal ID, Maughan TS, Smith CG, Cheadle JP, Fisher D, et al. Mismatch repair status and BRAF mutation status in metastatic colorectal cancer patients: a pooled analysis of the CAIRO, CAIRO2, COIN, and FOCUS studies. Clin Cancer Res. 2014;20(20):5322-30.

24 Kostakis ID, Agrogiannis G, Vaiopoulos AG, Mylona E, Patsouris E, Kouraklis G, et al. A clinicopathological analysis of KISS1 and KISS1R expression in colorectal cancer. APMIS. 2015;123(7):629-37.

25 Martínez-Pérez J, Lopez-Calderero I, Saez C, Benavent M, Limon ML, Gonzalez-Exposito $\mathrm{R}$, et al. Prognostic relevance of Src activation in stage II-III colon cancer. Hum Pathol. 2017;67:119-25.

26 Al-Maghrabi J, Emam E, Gomaa W, Saggaf M, Buhmeida A, Al-Qahtani M, et al. c-MET immunostaining in colorectal carcinoma is associated with local disease recurrence. BMC Cancer. 2015;15:676

27 Martini G, Cardone C, Vitiello PP, Belli V, Napolitano S, Troiani T, et al. EPHA2 Is a Predictive Biomarker of Resistance and a Potential Therapeutic Target for Improving Antiepidermal Growth Factor Receptor Therapy in Colorectal Cancer. Mol Cancer Ther. 2019; 18(4):845-55.

28 Jang BG, Kim HS, Chang WY, Bae JM, Kim WH, Kang GH. Expression Profile of LGR5 and Its Prognostic Significance in Colorectal Cancer Progression. Am J Pathol. 2018; 188(10):2236-50.

29 Sebio A, Matsusaka S, Zhang W, Yang D, Ning Y, Stremitzer S, et al. Germline polymorphisms in genes involved in the Hippo pathway as recurrence biomarkers in stages II/III colon cancer. Pharmacogenomics J. 2016;16(4):312-9.

30 Greally M, Kelly CM, Cercek A. HER2: An emerging target in colorectal cancer. Curr Probl Cancer. 2018;42(6):560-71.

31 Raghav KPS, Overman MJ, Yu R, MericBernstam F, Menter D, Kee BK, et al. HER2 amplification as a negative predictive biomarker for anti-epidermal growth factor receptor antibody therapy in metastatic colorectal cancer. JCO. 2016;34(15_Suppl 1):3517. 
32 Nam SK, Yun S, Koh J, Kwak Y, Seo AN, Park $\mathrm{KU}$, et al. BRAF, PIK3CA, and HER2 Oncogenic Alterations According to KRAS Mutation Status in Advanced Colorectal Cancers with Distant Metastasis. PLoS One. 2016; 11(3):e0151865.

33 Pietrantonio F, Di Nicolantonio F, Schrock $\mathrm{AB}$, Lee J, Morano F, Fucà G, et al. RET fusions in a small subset of advanced colorectal cancers at risk of being neglected. Ann Oncol. 2018;29(6):1394-401.

34 Björkman K, Mustonen H, Kaprio T, Haglund C, Böckelman C. Mucin 16 and kallikrein 13 as potential prognostic factors in colon cancer: Results of an oncological 92-multiplex immunoassay. Tumour Biol. 2019; 41(7): 1010428319860728.

35 Ahadi M, Andrici J, Sioson L, Sheen A, Clarkson A, Gill AJ. Loss of Hes1 expression is associated with poor prognosis in colorectal adenocarcinoma. Hum Pathol. 2016;57:91-7.

36 Meng H, Li W, Boardman LA, Wang L. Loss of ZG16 is associated with molecular and clinicopathological phenotypes of colorectal cancer. BMC Cancer. 2018;18(1):433.

37 Lee LH, Cavalcanti MS, Segal NH, Hechtman JF, Weiser MR, Smith JJ, et al. Patterns and prognostic relevance of PD-1 and PD-L1 expression in colorectal carcinoma. Mod Pathol. 2016;29(11):1433-42.
38 Wyss J, Dislich B, Koelzer VH, Galván JA, Dawson H, Hädrich M, et al. Stromal PD-1/ PD-L1 Expression Predicts Outcome in Colon Cancer Patients. Clin Colorectal Cancer. 2019;18(1):e20-e38.

39 van der Waals LM, Borel Rinkes IHM, Kranenburg O. ALDH1A1 expression is associated with poor differentiation, 'right-sidedness' and poor survival in human colorectal cancer. PLoS One. 2018;13(10):e0205536.

40 Ichimura N, Shinjo K, An B, Shimizu Y, Yamao K, Ohka F, et al. Aberrant TET1 Methylation Closely Associated with CpG Island Methylator Phenotype in Colorectal Cancer. Cancer Prev Res (Phila). 2015;8(8):702-11.

41 Pietrantonio F, Di Nicolantonio F, Schrock AB, Lee J, Tejpar S, Sartore-Bianchi A, et al. ALK, ROS1, and NTRK Rearrangements in Metastatic Colorectal Cancer. J Natl Cancer Inst. 2017;109:10.

42 Eto T, Miyake K, Nosho K, Ohmuraya M, Imamura $Y$, Arima K, et al. Impact of loss-offunction mutations at the RNF43 locus on colorectal cancer development and progression. J Pathol. 2018;245(4):445-55.
43 Böhm J, Muenzner JK, Caliskan A, Ndreshkjana B, Erlenbach-Wünsch K, Merkel S, et al. Loss of enhancer of zeste homologue 2 (EZH2) at tumor invasion front is correlated with higher aggressiveness in colorectal cancer cells. J Cancer Res Clin Oncol. 2019; 145(9):2227-40.

44 Yamamoto I, Nosho K, Kanno S, Igarashi $\mathrm{H}$, Kurihara $\mathrm{H}$, Ishigami K, et al. EZH2 expression is a prognostic biomarker in patients with colorectal cancer treated with anti-EGFR therapeutics. Oncotarget. 2017;8(11):178108.

45 Bach S, Sluiter NR, Beagan JJ, Mekke JM, Ket JCF, van Grieken NCT, et al. Circulating Tumor DNA Analysis: Clinical Implications for Colorectal Cancer Patients. A Systematic Review. JNCI Cancer Spectr. 2019;3(3):pkz042.

46 Nicolazzo C, Raimondi C, Gradilone A, Emiliani A, Zeuner A, Francescangeli F, et al. Circulating Tumor Cells in Right- and Left-Sided Colorectal Cancer. Cancers. 2019;11(8):1042.

$47 \mathrm{Hu} \mathrm{Y,} \mathrm{Guo} \mathrm{H,} \mathrm{Chen} \mathrm{Y,} \mathrm{Chen} \mathrm{Y,} \mathrm{Lan} \mathrm{L,} \mathrm{Li} \mathrm{J,} \mathrm{et}$ al. The mutational landscape of circulating cell free DNA in patients with colorectal adenoma and carcinoma. JCO. 2018;36(15 Suppl l):e15662. 